\title{
Harmonisation of in-silico next-generation sequencing based methods for diagnostics and surveillance
}

\section{J. Nunez-Garcia}

Animal and Plant Health Agency

\section{AbuOun}

Animal and Plant Health Agency

\section{N. Storey}

Animal and Plant Health Agency

\section{Brouwer}

Wageningen University \& Research

J.F Delgado-Blas

Universidad Complutense de Madrid

\section{S. S. Mo}

Norwegian Veterinary Institute

N. Ellaby

Public Health England

\section{K. T. Veldman}

Wageningen University \& Research

\section{Haenni}

Unité Antibiorésistance et Virulence Bactériennes, (Anses)

\section{P. Châtre}

Unité Antibiorésistance et Virulence Bactériennes, (Anses)

\section{J. Y. Madec}

Unité Antibiorésistance et Virulence Bactériennes, (Anses)

\section{J. A. Hammerl}

German Federal Institute for Risk Assessment

C. Serna

Universidad Complutense de Madrid

M. Getino

University of Surrey

R. LaRagione

University of Surrey

\section{T. Naas}

Assistance Publique Hopitaux De Paris

\section{A. A. Telke}

Norwegian Veterinary Institute

P. Glaser

Institute Pasteur

\section{Sunde}

Norwegian Veterinary Institute

\section{B. Gonzalez-Zorn}

Universidad Complutense de Madrid

M. J. Ellington

Public Health England

M. Anjum ( muna.anjum@apha.gov.uk) 
Animal and Plant Health Agency

\section{Research Article}

Keywords:

Posted Date: February 17th, 2022

DOI: https://doi.org/10.21203/rs.3.rs-1351139/v1

License: () (1) This work is licensed under a Creative Commons Attribution 4.0 International License. Read Full License 


\section{Abstract}

Improvements in cost and speed of next generation sequencing (NGS) have provided a new pathway for delivering disease diagnosis, molecular typing, and detection of antimicrobial resistance (AMR). Numerous published methods and protocols exist, but a lack of harmonisation has hampered meaningful comparisons between results produced by different methods/protocols vital for global genomic diagnostics and surveillance. As an exemplar, this study evaluated the sensitivity and specificity of five well-established insilico AMR detection software, produced from whole genome sequencing a panel of 438 Escherichia coli. No significant differences in software performance were observed, suggesting that efforts to harmonise AMR predictions from sequence data should focus on: 1) establishing universal minimum levels of performance thresholds (e.g. a control isolate panel, minimum sensitivity/specificity thresholds); 2) standardising AMR gene identifiers in reference databases and gene nomenclature; 3) producing consistent genotype/phenotype correlations. The study also revealed limitations of in-silico technology on detecting resistance to certain antimicrobials due to lack of specific fine-tuning options in bioinformatics tool or a lack of representation of resistance mechanisms in the reference database. Lastly, we noted user friendliness of tools was also an important consideration. Therefore, our recommendations are timely for widespread standardisation of bioinformatics for genomic diagnostics and surveillance globally.

\section{Background}

Next Generation Sequencing (NGS), a DNA sequencing technology, has become an established technique with hundreds of publications each year detailing the use and advancement of this technology. Furthermore, NGS high-throughput platforms, which in recent years have seen radical improvements in quality, running times and cost, have revolutionised the diagnosis of health-related issues in animals and humans. This includes infectious disease diagnosis, where in-silico genetic data analysis is aiding and, in some cases, substituting more complex and costly laboratory techniques ${ }^{1}$. The SARS-CoV-19 pandemic is a testimony of the usefulness of this technology for both research and surveillance ${ }^{2,3}$. By sequencing the whole genome of bacterial isolates, using NGS technologies, transmission chains of pathogens and a global overview of their population structure is being identified, helping trace outbreaks ${ }^{4}$. For antimicrobial resistance (AMR) detection in bacteria, which is another global threat leading to decreasing therapeutic options and increasing treatment failures, antimicrobial susceptibility testing which produces a phenotype, is progressively being substituted by detection of the underlying genetic mechanisms using whole genome sequencing (WGS) of bacterial isolates. WGS analysis facilitates the identification of bacteria such as Escherichia coli, their lineage, and genetic features such as the gain of AMR genes, including resistance to antimicrobials critically important for therapeutics, and chromosomal mutations, deletions and insertions that may be associated with AMR phenotypes ${ }^{5-10}$.

However, a major barrier to the application of bioinformatics software for AMR detection beyond individual research applications to diagnosis and national and/or international surveillance, is the standardisation of both DNA-based laboratory techniques and in-silico analysis. The availability of a plethora of bioinformatics tools and pipelines, with continual rapid advancement in this area, has resulted in no standardised methodology or nomenclature making comparisons across compartments (e.g. humans, animals, and environment) or institutes difficult. In 2019, Hendriksen et al ${ }^{11}$ reported at least 47 bioinformatics tools were freely available, and no doubt this has increased further. However, to understand the changing epidemiology of AMR in an One-Health context for monitoring evolution of AMR genes and bacteria, so appropriate control measures can be in place, it is vital to harmonise in silico AMR detection methods, as has been established for bacteria such as MRSA, where ideal requirements for molecular typing techniques have been clearly defined ${ }^{12,13}$. There have been similar discussions for in silico AMR detection ${ }^{14}$, and although the recommendations have not been properly evaluated, a small multi-centre study with nine institutes that performed predictions of AMR genotypes from 10 samples harbouring carbapenem-resistant organisms, showed that differences in the database selected and gene coverage thresholds were some of the factors contributing to variation in AMR results ${ }^{15}$. Such evaluations are required at a much larger scale because supranational organisations such as the World Health Organisation (WHO), European Centre for Disease Control (ECDC) and the European Food Safety Authority (EFSA) have recommended the use of genomics within international surveillance programmes that compare AMR trends across countries in Europe and worldwide, to help tackle the spread of multi- and extensive drug resistant bacteria which are the cause of great concern ${ }^{16-18}$.

In silico AMR detection methods are based on a three-step process: i) sample preparation such as bacterial culture and DNA extraction; ii) whole genome sequencing; and iii) in-silico data analysis. This process offers attractive possibilities for diagnostic test automation, including parallelising tests for multiple characteristics and even retrospective exploration for novel AMR genotypes 
without having to repeat steps i) or ii). AMR detection pipelines are based on existing knowledge of AMR genotype-phenotype associations ${ }^{19}$.

To detect the genotype or underlying genetic mechanism for resistance by screening the WGS data obtained from isolated bacteria, the bacterial DNA is compared against a reference set of DNA sequences, also known as a database (normally in FASTA or text-based format) containing the genotypes (i.e. AMR genes or point mutations) responsible for known AMR phenotypes.

Bacterial DNA can be compared against the database using two different techniques: either by mapping the WGS short-reads onto the reference DNA sequences in the database, or by basic local alignment search ${ }^{20}$ using the assembled genome contigs as a query against the database. While the first approach may be faster ( $<10$ mins for a single core computer) and straighter forward, it involves dealing with large raw data files (e.g., paired short-reads raw data files of up to 300 Mbytes for a 5 Mbase genome such as the Escherichia coli (used in this study)). The second method may require a longer running time ( 25 minutes) as the raw data files must be de novo assembled prior to comparison against the database. Many laboratories perform the assembly step as a routine for other purposes, so the extra running time might not be a burden. Once the assembly for the bacterial genome has been stored, re-running the pipeline to screen for a novel AMR gene should be a matter of a few seconds.

As part of the One-Health European Joint Programme Project ARDIG (Antibiotic Resistance Dynamics: the influence of geographic origin and management systems on resistance gene flows within humans, animals and the environment) ${ }^{21}$, nine partners compared the performance of five software pipelines for AMR detection based on WGS of $E$. coli isolates and this paper describes the work. This study restricted its scope to comparing the performance of pipelines in terms of their sensitivity and specificity to detect AMR, under the default settings defined by the pipelines' authors. No attempt to evaluate the software installation process was considered in this study since most of the users agreed that IT support is provided institutionally. The DNA extraction and sequencing protocols from the participating institutes were also not evaluated in this study.

\section{Materials And Methods}

\section{Pipelines}

A description of the AMR detection software used in this study have been provided:

GeneFinder. Public Health England (PHE), UK ${ }^{24}$. URL: https://github.com/phe-bioinformatics/gene_finder. Operator. PHE. Language: python 2.7.5. Input format: FASTQ. Algorithm: mapping (bowtie 2.1.0). Reference database: provides three in house references sets in FASTA format for E.coli, Salmonella and Campylobacter. Users can incorporate their own reference set. Detection: presence or absence of sequences and mutations. It also reports insertions, deletions, mixed positions and large indels. Possibility to set the similarity thresholds (between sample DNA and a reference DNA) individually for each gene. Quality metrics: coverage, similarity, depth and coverage distribution.

APHA SeqFinder/ABRicate. Animal and Plant Health Agency (APHA), UK ${ }^{25}$. URL: https://github.com/APHA-AMR-VIR/APHASeqFinder. Operator: APHA. Language: python 3. Input format: FASTQ. Algorithm: mapping (smalt 0.7.6). Reference database: provides three in house reference sets in FASTA format for AMR genes, mutations and entero plasmids, virulence factors and heavy metal resistance. Detection: presence or absence of sequences and mutations. Quality metrics: coverage, similarity, depth and normalised depth by MLST genes. ABRicate [19] is used in conjunction with SeqFinder as an additional filter.

URL: https://github.com/tseemann/abricate. Language: perl. Input format: FASTA assembled contigs. (SPAdes 3.13.1). Algorithm: BLAST 2.7.0 or higher. Reference database: same references as used for APHA SeqFinder (it also provides additional databases; NCBI, CARD, ARG-ANNOT, ResFinder, MEGARES, EcOH, PlasmidFinder, Ecoli_VF and VFDB). Detection: presence or absence of genes. Quality metrics: coverage and similarity

BLAST, Wageningen Bioveterinary Research (WBVR), The Netherlands. Pipeline not published at the time of this study. Operator: WBVR. Input format: FASTA assembled contigs. Algorithm: raw reads are error corrected with Tadpole from the BBduk suite v38.71. Quality trimming to Q20 with BBduk. Genomes are assembled using SPAdes 3.13.1. Assemblies are compared to the reference database using BLAST (with filters: $98 \%$ sequence identity and 97\% gene coverage). Reference database: ResFinder database used. Detection: presence or absence of sequences and mutations. Quality metrics: sequence identity and gene coverage provided by BLAST. 
ResFinder + PointFinder. ${ }^{26,27}$ Technical University of

Denmark. URL: https://bitbucket.org/genomicepidemiology/resfinder/src/master/. Operator. The Norwegian Veterinary Institute (NVI). Language: python 3. Input format: FASTQ or FASTA assembled contigs. Algorithm: BLAST is used to analyse assemblies (FASTA files). Mapper KMA is used to analyse read data (FASTQ files). Reference database: RestFinder database and PointFinder_database (they cover several bacteria). Detection: presence or absence of sequences and mutations. Quality metrics:

ARIBA ${ }^{28}$, Sanger Institute, UK. URL: https: //github.com/sanger-pathogens/ariba. Operator: Universidad Complutense de Madrid (UCM). Language: python 3. Input format: FASTQ. Algorithm: mapping (Bowtie 2.1.0). Reference database: does not provide its own reference database but has an integrated method to download and standardise one from different sources such as the Comprehensive Antibiotic Resistance Database (CARD), ResFinder, ARG-ANNOT, MEGARes, NCBI, PlasmidFinder, VFDB, SRST2 and VirulenceFinder. Users can incorporate their own reference set. Detection: presence or absence of sequences only (although it is possible to incorporate an external reference database for detecting mutations, currently, there is not an integrated and standardised database for mutations responsible for AMR). It also reports genetic fragmentations, interruptions, and duplications. Quality metrics: gene coverage, sequence identity.

The raw reads for isolates from this dataset are available in the NCBI nucleotide archive under project number PRJNA805266.

\section{The antimicrobials}

The evaluation was performed on the 14 antimicrobials used for AMR monitoring by the European Food and Safety (EFSA) [4/115]: Ampicillin, Azithromycin, Cefotaxime, Ceftazidime, Chloramphenicol, Ciprofloxacin, Colistin, Gentamicin, Meropenem, Nalidixic Acid, Sulfamethoxazole, Tetracycline, Tigecyline and Trimethoprim. The susceptibility of wild type $E$. coli to the panel of 14 antimicrobials was tested, and were categorised as susceptible or resistant by: susceptible, a bacterial isolate was defined as susceptible when it is inhibited at a concentration of a specific antimicrobial equal or lower than the established cut-off value; and resistant, a bacterial isolate was defined as resistant when it is not inhibited at a concentration of a specific antimicrobial higher than the established cutoff value following the ECOFF values for the MIC described by the EUCAST ${ }^{23}$.

\section{The isolates}

A total of 438 E. coli isolates from were provided by 9 European institutes. The French Agency for Food, Environmental and Occupational Health and Safety, Lyon - France (49 isolates). Universidad Complutense de Madrid, Spain (50 isolates). Institute Pasteur, France (52 isolates). The German Federal Institute for Risk Assesment, Germany (50 isolates). The Norwegian Veterinary Institute, Norway (50 isolates). Wageningen Bioveterinary Research, The Netherlands (50 isolates). The University of Surrey, United Kingdom (50 isolates). Animal and Plant Health Agency, United Kingdom (37 isolates). Public Health England, United Kingdom (51 isolates).

\section{Data analysis}

The institutes provided the WGS data and the MIC values established using the EFSA protocol. In Table S1, a summary of the number of isolates per institute is provided. Also, the number (and percentage) of isolates presenting resistance (res) and susceptibility (sen) to each of the 14 antimicrobials is given. For some institutions-antimicrobial, MIC values were not available for part or all the isolates, this has been marked with an asterisk. Most cases from human samples (PHE) for which some antimicrobials not affecting humans are not routinely screened (azithromycin, chloramphenicol, sulfamethoxazole, tetracycline and trimethoprim). Note that for some antibiotics, institutes were unable to provide isolates showing resistance.

Analysis for all 438 isolates was performed by five independent operators (one per pipeline). Result tables from the pipeline runs were sent to each of the nine donor institutes who extracted their corresponding isolates results for all five pipelines. The information was recorded on a standardised form composed of 14 sub-tables; one reporting for each isolate (rows), the gold standard classification (resistance or sensitivity using the ECOFF interpretation) of the MIC values and the AMR elements (genes or chromosomal mutations) detected for each of the 14 antimicrobials.

For this study, values in the tables were interpreted as ones or zeroes depending on whether any AMR genotype was detected for each isolate-antimicrobial cell. These values were then compared to the gold standard (ECOFF interpretation of the MIC values) to calculate the sensitivity and specificity and their $95 \%$ confidence intervals (shown is Table 2 ).

Page 5/15 


\section{Results}

\section{Antimicrobial sensitivity of $E$. coli}

A total of $438 \mathrm{E}$. coli collected by nine different collaborating institutes working in the veterinary and human health sectors in Europe, were included in the study. The antimicrobial susceptibilities of all isolates were established to the panel of 14 EFSA antimicrobials using a standard protocol ${ }^{22}$, and antimicrobial resistance was evaluated by the Epidemiological Cut-Off (ECOFF) values from the minimum inhibitory concentration (MIC), as described by the European Committee on Antimicrobial Susceptibility Testing (EUCAST) ${ }^{23}$. The interpretation derived from the MIC values by applying ECOFFs to wild type isolates was used as the gold standard for this study. The total number of isolates assayed by MIC per antimicrobial by each institute has been given in Table 1 .

Our panel of isolates did not show an even distribution of resistance for all 14 antimicrobials, although for most antimicrobials the numbers of resistant isolates in comparison to sensitive ones was more than $10 \%$ of the total, so will enable accurate sensitivity estimates to be made (Table 1). However, smaller numbers of isolates were determined as resistant to colistin $(n=27$ or $6.2 \%)$ and tigecycline ( $n=1$ or $0.23 \%$ ), consistent with the scarcity of $E$. coli resistant to these last resort critically important antimicrobials (HP$\mathrm{CIAs}$ ) in Europe. The unbalanced ratio between resistant and susceptible isolates for these antimicrobials will result in loss of precision for the sensitivity estimates. Nevertheless, sensitivity estimates for colistin was still calculated but for tigecycline, the sensitivity was not calculated due to the very low numbers of resistant isolates.

\section{Pipeline comparisons}

Five pipelines were selected for testing: GeneFinder ${ }^{24}$; APHA SeqFinder/Abricate ${ }^{25}$; WBVR BLAST (in-house pipeline); ResFinder/PointFinder ${ }^{26,27}$; ARIBA ${ }^{28}$. To avoid operator bias with respect to possible variations in the pipeline settings and results interpretation, five independent operators (one per pipeline) ran all 438 isolates using default settings, then results from each pipeline were sent to the nine donor institutes. Using pre-set interpretation criteria (see Methods for detail), each institute collated the results for their isolates with the corresponding MIC data into a standardised format. Estimates of sensitivity and specificity for each antimicrobial compared to the phenotypic data per isolate for each pipeline are shown in Table 2. The cells have been coloured depending on the different levels of performance. This should be interpreted with care as there is significant overlap between the confidence intervals from different pipelines, as demonstrated by Figure 1, with plots showing the sensitivity, specificity and the $95 \%$ confidence intervals for different antimicrobials on the receiver operating characteristic (ROC) coordinate system. All pipelines had an overall sensitivity value between 0.9 and 0.95 when comparing the genotype with phenotype for each antimicrobial class (Table 2A) except for ARIBA, due to this pipeline only reporting the presence or absence of acquired resistance genes and not including resistance associated with chromosomal point mutations that reduce susceptibility to antimicrobials such as fluroquinolones ${ }^{19}$. The average specificity value, when comparing the genotype with phenotype for each antimicrobial class for all pipelines, (Table 2B) was around 0.89 , except for the APHA Seqfinder/ABRicate pipeline that showed a slightly higher value at 0.93 . This is probably due to it combining two stages: a discovery stage which applies the APHA Seqfinder software, and a validation stage using ABRicate. From the sensitivity and specificity estimates, all the pipelines, in general terms, showed similar levels of performance.

Resistance to several antimicrobials were easier to detect, such as to ampicillin and tetracycline, with average sensitivity/specificity values equal to $0.95 / 0.96$ and 0.97/0.93, respectively (Table 2 and Figure 1). Colistin resistance was the most difficult to detect with average sensitivity/specificity values of $0.70 / 0.99$ (Table 2 and Figure 1). This difficulty can be attributed to two possible factors: the genotype associated to the colistin AMR phenotype might not be fully represented in the database, i.e., there may be an unknown gene or chromosomal mutation that has not yet been associated to this phenotype; and/or the thresholds used in the pipelines for AMR gene detection might be suboptimal for this antimicrobial. Commonly used thresholds for AMR detection in some pipelines include the percentage of an AMR gene present within the isolate and the percentage of similarity between the reference AMR gene and the test isolate. By decreasing or increasing the thresholds, it will be possible to trade off the sensitivity and specificity values, and hence to adjust the detection capabilities. Relaxing the pipeline thresholds will increase the sensitivity and decrease the false negative rate (type II error) causing a decrease of the specificity and the consequent increase of the false positive rate (type I error). Thus, being able to input the pipeline thresholds may be an important feature to adjust the balance between sensitivity and specificity values. Further, this adjustment should be individually defined for each of the antimicrobial classes represented in the database, since a set of thresholds that may be optimal for the detection of one antimicrobial resistance might produce poor results for another. The 
GeneFinder pipeline was the only pipeline in this study that allowed the user to set individual similarity threshold for each database entry.

Optimum threshold values might also depend on the specific purpose for detecting resistance. In some cases, a very high sensitivity may be preferred as a trade-off to lowering the specificity. For example, more relaxed thresholds for colistin, which belongs to the HPCIA list ${ }^{29}$, may be used to minimise the occurrence of undetected resistance.

\section{Interpretation of pipeline results}

While performance of the pipeline is the major factor when deciding which one is most suitable to user needs, the ease of interpreting the results files is also an important point to consider. To this end, a questionnaire was prepared and completed by operators/participants from each of the nine institutes. The questionnaire contained seven subjective questions to measure the degree of user friendliness related to the interpretation of the pipeline output (see Table 3 for details of the questions and the average values for each question and pipeline given in response by each operator). There were no major differences found between the pipelines, with the APHA Seqfinder/ABRicate having the highest mark and ARIBA the lowest. The preferred pipelines (as per question 7) was GeneFinder by a small margin over APHA Seqfinder/ABRicate. Therefore, user friendliness of tools should also be considered as part of any pipeline harmonisation process, as it may influence who and how often tools are used.

\section{Discussion}

Standardisation of any methodology is essential to enable comparison, as well as reproducibility across different sectors and countries, but can often be undervalued or overlooked. Monitoring systems that are harmonised already exists in areas such as AMR, which has been invaluable to determine AMR trends overtime across Europe although it only uses phenotypic testing results ${ }^{30}$. The wealth of data WGS provides and the increased cost effectiveness of NGS technology has presented genomic epidemiology as a feasible alternative. However, the availability and continual development of new bioinformatics tools has resulted in a call for harmonisation of in silico genomic methods to track AMR globally ${ }^{11}$. Recommendations made in a workshop to implement WGS for surveillance recognised the challenges facing its implementation, including some of the bioinformatics processes ${ }^{14}$, which was the focus of this study. Although some of the participating institutes for this study extensively use their preferred in-silico methodologies for AMR detection, in addition to classical wet-lab techniques, the global harmonisation of phenotype-genotype AMR susceptibilities is still in its early stages, due to a lack pre-set standards. Here we compared several AMR bioinformatics pipelines using the same isolate data set, with default (generalised) pipeline settings and under the same interpretation conditions. Using these conditions we concluded that no pipeline clearly stands out from the rest, in terms of performance and ease of output interpretation. Further, we observed that the performance of the pipelines depends on the antimicrobial for which the resistance determinant was being detected and therefore the ability to set individual thresholds for each database entry is an important feature.

We believe that the results of our study can be applied to inform future initiatives for harmonisation of results from WGS pipelines, whether for AMR or any other area for diagnostics and surveillance. Just as multi-locus sequence typing of $E$. coli using underlying genetics $^{4}$ is increasingly being used in place of serotyping ${ }^{31}$ to identify pathogens due to its ability to provide more detailed/accurate subtyping of populations, we believe a harmonised WGS method will do the same for bacterial characterisation, including for AMR. Therefore, our recommendations for harmonisation are as follows. Firstly, it may not be relevant which pipeline is used as long as it verifies a certain level of performance that can be agreed by the relevant scientific experts, depending on the application and the establishment of common inclusion/exclusion criteria of targeted matches. We propose that a control set of isolates are used to test and evaluate any pipeline with an appropriate representative sample and pre-set validation thresholds. For example, the collection of isolates used in this study may be appropriate for testing in silico AMR pipelines, although any well validated set may be included, provided there is a balanced ratio between resistance and susceptibility of isolates to antimicrobials included in the test panel, e.g., to the EFSA panel of antimicrobials. However, the isolate panel will need to be regularly updated to incorporate isolates with new/novel AMR genes and the pipelines re-evaluated. Also, a certain level of pre-agreed performance in terms of minimum sensitivity and specificity thresholds, when comparisons are made between phenotypes and genotypes, should be used as a validation test for any AMR detection software. Although, for HPCIAs e.g. colistin or carbapenem a more relaxed threshold may be used to maximise resistance detection, including of new variants Secondly, we recommend unifying the databases used by different pipeline software for positive identification; unless genes present in databases, including their nomenclature, are harmonised, there will be differences in the output even from the same test set. In the AMR context, AMR gene identifiers or sequences, including any chromosomal point 
mutations leading to reduced susceptibility, and the translation rules from genotype to phenotype, should be consistent and transparent. This will also help the naïve user in interpretation of genotypic data, in addition to promoting harmonisation. And thirdly, to allow greater access and usability of this technology for routine surveillance, the final output information should be standardised into user-friendly documents. This will enable individuals with minimal background in genetics to benefit from these software.

As there are countless bioinformatics tools available, and many of them pursue similar aims but use different approaches with numerous fine tune adjustments, continual comparison of their performance is a difficult task. Our recommendation to achieve harmonisation does not require focusing on the best performing software, but on setting a common evaluation process based on universal minimal performance thresholds applied to a representative testing sample set. In other words, we have made recommendations which will help towards creation of an appropriate structure for global standardisation of the bioinformatics component to enable genomic surveillance and diagnostics to become routine and standardised worldwide.

\section{Declarations}

\section{Author contributions}

Conceptualisation: M.F.A., J. N-G., M.A., P.G, M.J.E, B.G-Z, M.S, R.L.R, M.S.B.

Methodology/Initial Analysis: M.A., N.S., M.S.B, J.F. D-B, M.S.S., N.E, M.H, C.S., A.A.T., J.A.H., P.C.

Statistical Analysis of WGS data: J. N-G.

Writing - Original Draft Preparation: J. N-G., M.A. and M.F.A.

Writing - Review and Editing: all authors.

Funding: M.F.A., P.G., M.J.E, B.G-Z, M.S, R.L.R, M.S.B and J.A.H

\section{Data availability}

All WGS data is available through NCBI BioProject ID: PRJNA805266.

\section{Competing Interest Statement}

The authors declare that there are no conflicts of interest.

\section{Funding information}

Funding was from the European Union's Horizon 2020 research and innovation programme under Grant Agreement No 773830, in the ARDIG project within the One Health European Joint Programme; MFA also received funding from the Veterinary Medicines Directorate, UK, project VM0533.

\section{References}

1. Lefterova, M. I., Suarez, C. J., Banaei, N. \& Pinsky, B. A. Next-Generation Sequencing for Infectious Disease Diagnosis and Management: A Report of the Association for Molecular Pathology. J Mol Diagn 17, 623-634, doi:10.1016/j.jmoldx.2015.07.004 (2015).

2. Meredith, L. W. et al. Rapid implementation of SARS-CoV-2 sequencing to investigate cases of health-care associated COVID-19: a prospective genomic surveillance study. Lancet Infect Dis 20, 1263-1271, doi:10.1016/S1473-3099(20)30562-4 (2020).

3. Furuse, Y. Genomic sequencing effort for SARS-CoV-2 by country during the pandemic. Int J Infect Dis 103, 305-307, doi:10.1016/j.ijid.2020.12.034 (2021).

4. Zhou, Z. et al. The EnteroBase user's guide, with case studies on Salmonella transmissions, Yersinia pestis phylogeny, and Escherichia core genomic diversity. Genome Res 30, 138-152, doi:10.1101/gr.251678.119 (2020).

5. Liu, X., Thungrat, K. \& Boothe, D. M. Occurrence of OXA-48 Carbapenemase and Other $\beta$-Lactamase Genes in ESBL-Producing Multidrug Resistant Escherichia coli from Dogs and Cats in the United States, 2009-2013. Front Microbiol 7, 1057, 
doi:10.3389/fmicb.2016.01057 (2016).

6. Ewers, C. et al. Genomic Diversity and Virulence Potential of ESBL- and AmpC- $\beta$-Lactamase-Producing Escherichia coli Strains From Healthy Food Animals Across Europe. Front Microbiol 12, 626774, doi:10.3389/fmicb.2021.626774 (2021).

7. Schaufler, K. et al. Genomic and Functional Analysis of Emerging Virulent and Multidrug-Resistant Escherichia coli Lineage Sequence Type 648. Antimicrobial agents and chemotherapy 63, doi:10.1128/aac.00243-19 (2019).

8. AbuOun, M. et al. Characterizing Antimicrobial Resistant Escherichia coli and Associated Risk Factors in a Cross-Sectional Study of Pig Farms in Great Britain. Front Microbiol 11, 861, doi:10.3389/fmicb.2020.00861 (2020).

9. Duggett, N. A. et al. Occurrence and characterization of $m c r-1$-harbouring Escherichia coli isolated from pigs in Great Britain from 2013 to 2015. J Antimicrob Chemother 72, 691-695, doi:10.1093/jac/dkw477 (2017).

10. Duggett, N. et al. The importance of using whole genome sequencing and extended spectrum beta-lactamase selective media when monitoring antimicrobial resistance. Sci Rep 10, 19880, doi:10.1038/s41598-020-76877-7 (2020).

11. Hendriksen, R. S. et al. Using Genomics to Track Global Antimicrobial Resistance. Front Public Health 7, 242, doi:10.3389/fpubh.2019.00242 (2019).

12. Stefani, S. et al. Meticillin-resistant Staphylococcus aureus (MRSA): global epidemiology and harmonisation of typing methods. Int J Antimicrob Agents 39, 273-282, doi:10.1016/j.ijantimicag.2011.09.030 (2012).

13. Friedrich, A. W. et al. A European laboratory network for sequence-based typing of methicillin-resistant Staphylococcus aureus (MRSA) as a communication platform between human and veterinary medicine-an update on SeqNet.org. Euro Surveill 13 (2008).

14. Angers-Loustau, A. et al. The challenges of designing a benchmark strategy for bioinformatics pipelines in the identification of antimicrobial resistance determinants using next generation sequencing technologies. F1000Res 7, doi:10.12688/f1000research.14509.2 (2018).

15. Doyle, R. M. et al. Discordant bioinformatic predictions of antimicrobial resistance from whole-genome sequencing data of bacterial isolates: an inter-laboratory study. Microb Genom 6, doi:10.1099/mgen.0.000335 (2020).

16. Authority, E. F. S. Antimicrobial resistance shows no signs of slowing down.. (2019).

17. European Food SAfety AuthorityEuropean Food Safety Authority. Antimicrobial resistance in the EU: infections with foodborne bacteria becoming harder to treat. (2020) https://www.efsa.europa.eu/en/news/antimicrobial-resistance-eu-infections-foodbornebacteria-becoming-harder-treat.

18. Organisation, W. H. Global Antimicrobial Resistance and Use Surveillance System (GLASS) Whole-genome sequencing for surveillance of antimicrobial resistance. (2020).

19. Stubberfield, E. et al. Use of whole genome sequencing of commensal Escherichia coli in pigs for antimicrobial resistance surveillance, United Kingdom, 2018. Euro Surveill 24, doi:10.2807/1560-7917.ES.2019.24.50.1900136 (2019).

20. Altschul, S. F., Gish, W., Miller, W., Myers, E. W. \& Lipman, D. J. Basic local alignment search tool. J Mol Biol 215, 403-410, doi:10.1016/S0022-2836(05)80360-2 (1990).

21. Anonymous. One Health European Joint Programme. Antibiotic Resistance Dynamics: the influence of geographic origin and management systems or resistance gene flows within humans, animals and the environment (ARDIG). https://onehealthejp.eu/jrp-ardig/ (2020).

22. European Food SAfety Authority. Guidance on the assessment of bacterial susceptibility to antimicrobials of human and veterinary importance. EFSA Panel on Additives and Products or Substances used in Animal Feed (FEEDAP). EFSA Journal 10, 2740 (2012).

23. The European Committee on Antimicrobial Susceptibility Testing -EUCAST,. Antimicrobial wild type distributions of microorganisms. (2021) https://mic.eucast.org/.

24. Neuert, S. et al. Prediction of Phenotypic Antimicrobial Resistance Profiles From Whole Genome Sequences of Non-typhoidal Salmonella enterica. Front Microbiol 9, 592, doi:10.3389/fmicb.2018.00592 (2018).

25. AbuOun, M. et al. A genomic epidemiological study shows that prevalence of antimicrobial resistance in Enterobacterales is associated with the livestock host, as well as antimicrobial usage. Microb Genom 7, doi:10.1099/mgen.0.000630 (2021).

26. Bortolaia, V. et al. ResFinder 4.0 for predictions of phenotypes from genotypes. J Antimicrob Chemother 75, 3491-3500, doi:10.1093/jac/dkaa345 (2020). 
27. Zankari, E. et al. PointFinder: a novel web tool for WGS-based detection of antimicrobial resistance associated with chromosomal point mutations in bacterial pathogens. J Antimicrob Chemother 72, 2764-2768, doi:10.1093/jac/dkx217 (2017).

28. Hunt, M. et al. ARIBA: rapid antimicrobial resistance genotyping directly from sequencing reads. Microb Genom 3, e000131, doi:10.1099/mgen.0.000131 (2017).

29. Organization, W. H. Critically important antimicrobials for human medicine: 6th revision.. (2019).

30. European Food Safety Authority. Technical specifications on harmonised monitoring of antimicrobial resistance in zoonotic and indicator bacteria from food-producing animals and food. EFSA J 17, e05709, doi:10.2903/j.efsa.2019.5709 (2019).

31. Anjum, M. F., Tucker, J. D., Sprigings, K. A., Woodward, M. J. \& Ehricht, R. Use of miniaturized protein arrays for Escherichia coli 0 serotyping. Clin Vaccine Immunol 13, 561-567, doi:10.1128/CVI.13.5.561-567.2006 (2006).

\section{Tables}

Table 1. Number of resistant and susceptible isolates per antibiotic used in this study. Number of resistant and susceptible isolates provided by the nine collaborators institutes for each of the 14 antibiotics. For some institutions, for some antibiotics, MIC values were not made available for part or all the isolates, this has been marked with an $\left(^{*}\right)$. Cells in grey indicate unbalanced ratio with resistant isolates being less than $10 \%$ of total. 


\begin{tabular}{|c|c|c|c|c|}
\hline Institute & \# Strains & $\begin{array}{l}\text { Ampicillin } \\
\text { (res/sen) }\end{array}$ & $\begin{array}{l}\text { Azithromycin } \\
\text { (res/sen) }\end{array}$ & $\begin{array}{l}\text { Cefotaxime } \\
\text { (res/sen) }\end{array}$ \\
\hline APHA & 37 & $33(89.19 \%) / 4(10.81 \%)$ & $7(18.92 \%) / 30(81.08 \%)$ & $17(45.95 \%) / 20(54.05 \%)$ \\
\hline BfR & 50 & $41(82.0 \%) / 9(18.0 \%)$ & $5(10.0 \%) / 45(90.0 \%)$ & $28(56.0 \%) / 22(44.0 \%)$ \\
\hline ANSES & 49 & $49(100.0 \%) / 0(0.0 \%)$ & $10(20.41 \%) / 39(79.59 \%)$ & $49(100.0 \%) / 0(0.0 \%)$ \\
\hline UCM & 50 & $39(78.0 \%) / 11(22.0 \%)$ & $3(6.0 \%) / 47(94.0 \%)$ & $0(0.0 \%) / 50(100.0 \%)$ \\
\hline Uos & 50 & $49(98.0 \%) / 1(2.0 \%)$ & $32(64.0 \%) / 18(36.0 \%)$ & $47(94.0 \%) / 3(6.0 \%)$ \\
\hline Pasteur & 52 & $44(84.62 \%) / 8(15.38 \%)$ & $16(30.77 \%) / 36(69.23 \%)$ & $28(53.85 \%) / 24(46.15 \%)$ \\
\hline WBVR & 50 & $32(64.0 \%) / 18(36.0 \%)$ & $0(0.0 \%) / 50(100.0 \%)$ & $24(48.0 \%) / 26(52.0 \%)$ \\
\hline PHE & 51 & $50(100.0 \%) / 0(0.0 \%)^{\star}$ & $0(0 \%) / 0(0 \%) *$ & $43(86.0 \%) / 7(14.0 \%)^{*}$ \\
\hline NVI & 50 & $44(88.0 \%) / 6(12.0 \%)$ & $0(0 \%) / 0(0 \%) *$ & $29(58.0 \%) / 21(42.0 \%)$ \\
\hline Total & 439 & $381(86.79 \%) / 57(12.98 \%)$ & $73(16.63 \%) / 265(60.36 \%)$ & $265(60.36 \%) / 173(39.41 \%)$ \\
\hline Institute & $\begin{array}{l}\text { Ceftazidime } \\
\text { (res/sen) }\end{array}$ & $\begin{array}{l}\text { Chloramphenicol } \\
\text { (res/sen) }\end{array}$ & $\begin{array}{l}\text { Ciprofloxacin } \\
\text { (res/sen) }\end{array}$ & $\begin{array}{l}\text { Colistin } \\
\text { (res/sen) }\end{array}$ \\
\hline APHA & $16(43.24 \%) / 21(56.76 \%)$ & $18(48.65 \%) / 19(51.35 \%)$ & $22(59.46 \%) / 15(40.54 \%)$ & $0(0.0 \%) / 37(100.0 \%)$ \\
\hline BfR & $29(58.0 \%) / 21(42.0 \%)$ & $19(38.0 \%) / 31(62.0 \%)$ & $29(58.0 \%) / 21(42.0 \%)$ & $18(36.0 \%) / 32(64.0 \%)$ \\
\hline ANSES & $43(87.76 \%) / 6(12.24 \%)$ & $27(55.1 \%) / 22(44.9 \%)$ & $36(73.47 \%) / 13(26.53 \%)$ & $7(14.29 \%) / 42(85.71 \%)$ \\
\hline UCM & $0(0.0 \%) / 50(100.0 \%)$ & $16(32.0 \%) / 34(68.0 \%)$ & $15(30.0 \%) / 35(70.0 \%)$ & $2(4.0 \%) / 48(96.0 \%)$ \\
\hline Uos & $45(90.0 \%) / 5(10.0 \%)$ & $3(6.0 \%) / 47(94.0 \%)$ & $44(88.0 \%) / 6(12.0 \%)$ & $0(0.0 \%) / 50(100.0 \%)$ \\
\hline Pasteur & $13(25.0 \%) / 39(75.0 \%)$ & $8(15.38 \%) / 44(84.62 \%)$ & $20(38.46 \%) / 32(61.54 \%)$ & $0(0.0 \%) / 52(100.0 \%)$ \\
\hline WBVR & $24(48.0 \%) / 26(52.0 \%)$ & $12(24.0 \%) / 38(76.0 \%)$ & $16(32.0 \%) / 34(68.0 \%)$ & $0(0.0 \%) / 50(100.0 \%)$ \\
\hline PHE & $41(82.0 \%) / 9(18.0 \%)^{\star}$ & $0(0 \%) / 0(0 \%) *$ & $50(100.0 \%) / 0(0.0 \%)^{\star}$ & $0(0.0 \%) / 50(100.0 \%)^{*}$ \\
\hline NVI & $29(58.0 \%) / 21(42.0 \%)$ & $11(22.0 \%) / 39(78.0 \%)$ & $30(60.0 \%) / 20(40.0 \%)$ & $0(0.0 \%) / 50(100.0 \%)$ \\
\hline Total & $240(54.67 \%) / 198(45.1 \%)$ & $114(25.97 \%) / 274(62.41 \%)$ & $262(59.68 \%) / 176(40.09 \%)$ & $27(6.15 \%) / 411(93.62 \%)$ \\
\hline Institute & $\begin{array}{l}\text { Gentamicin } \\
\text { (res/sen) }\end{array}$ & $\begin{array}{l}\text { Meropenem } \\
\text { (res/sen) }\end{array}$ & $\begin{array}{l}\text { Nalidixic Acid } \\
\text { (res/sen) }\end{array}$ & $\begin{array}{l}\text { Sulfamethoxazole } \\
\text { (res/sen) }\end{array}$ \\
\hline APHA & $8(21.62 \%) / 29(78.38 \%)$ & $0(0.0 \%) / 37(100.0 \%)$ & $20(54.05 \%) / 17(45.95 \%)$ & $32(86.49 \%) / 5(13.51 \%)$ \\
\hline BfR & $11(22.0 \%) / 39(78.0 \%)$ & $0(0.0 \%) / 50(100.0 \%)$ & $25(50.0 \%) / 25(50.0 \%)$ & $35(70.0 \%) / 15(30.0 \%)$ \\
\hline ANSES & $21(42.86 \%) / 28(57.14 \%)$ & $0(0.0 \%) / 49(100.0 \%)$ & $38(77.55 \%) / 11(22.45 \%)$ & $44(89.8 \%) / 5(10.2 \%)$ \\
\hline UCM & $6(12.0 \%) / 44(88.0 \%)$ & $0(0.0 \%) / 50(100.0 \%)$ & $28(56.0 \%) / 22(44.0 \%)$ & $36(72.0 \%) / 14(28.0 \%)$ \\
\hline Uos & $12(24.0 \%) / 38(76.0 \%)$ & $0(0.0 \%) / 50(100.0 \%)$ & $40(80.0 \%) / 10(20.0 \%)$ & $45(90.0 \%) / 5(10.0 \%)$ \\
\hline Pasteur & $12(23.08 \%) / 40(76.92 \%)$ & $23(44.23 \%) / 29(55.77 \%)$ & $21(40.38 \%) / 31(59.62 \%)$ & $40(76.92 \%) / 12(23.08 \%)$ \\
\hline WBVR & $0(0.0 \%) / 50(100.0 \%)$ & $0(0.0 \%) / 50(100.0 \%)$ & $8(16.0 \%) / 42(84.0 \%)$ & $26(52.0 \%) / 24(48.0 \%)$ \\
\hline PHE & $21(42.0 \%) / 29(58.0 \%)^{*}$ & $26(52.0 \%) / 24(48.0 \%)^{*}$ & $0(0 \%) / 0(0 \%) *$ & $0(0 \%) / 0(0 \%)^{\star}$ \\
\hline NVI & $7(14.0 \%) / 43(86.0 \%)$ & $0(0.0 \%) / 45(100.0 \%)^{*}$ & $18(36.0 \%) / 32(64.0 \%)$ & $29(58.0 \%) / 21(42.0 \%)$ \\
\hline
\end{tabular}




\begin{tabular}{llll}
\hline Institute & $\begin{array}{l}\text { Tetracycline } \\
\text { (res/sen) }\end{array}$ & $\begin{array}{l}\text { Tigecyline } \\
\text { (res/sen) }\end{array}$ & $\begin{array}{l}\text { Trimethoprim } \\
\text { (res/sen) }\end{array}$ \\
\hline APHA & $32(86.49 \%) / 5(13.51 \%)$ & $0(0.0 \%) / 37(100.0 \%)$ & $29(78.38 \%) / 8(21.62 \%)$ \\
\hline BfR & $38(76.0 \%) / 12(24.0 \%)$ & $0(0.0 \%) / 50(100.0 \%)$ & $33(66.0 \%) / 17(34.0 \%)$ \\
\hline ANSES & $42(85.71 \%) / 7(14.29 \%)$ & $0(0.0 \%) / 49(100.0 \%)$ & $36(73.47 \%) / 13(26.53 \%)$ \\
\hline UCM & $45(90.0 \%) / 5(10.0 \%)$ & $0(0.0 \%) / 50(100.0 \%)$ & $34(68.0 \%) / 16(32.0 \%)$ \\
\hline UoS & $28(56.0 \%) / 22(44.0 \%)$ & $0(0.0 \%) / 50(100.0 \%)$ & $38(76.0 \%) / 12(24.0 \%)$ \\
\hline Pasteur & $23(44.23 \%) / 29(55.77 \%)$ & $0(0.0 \%) / 52(100.0 \%)$ & $24(46.15 \%) / 28(53.85 \%)$ \\
\hline WBVR & $25(50.0 \%) / 25(50.0 \%)$ & $0(0.0 \%) / 50(100.0 \%)$ & $14(28.0 \%) / 36(72.0 \%)$ \\
\hline PHE & $0(0 \%) / 0(0 \%)^{*}$ & $1(2.0 \%) / 49(98.0 \%)^{*}$ & $0(0 \%) / 0(0 \%)^{*}$ \\
\hline NVI & $31(62.0 \%) / 19(38.0 \%)$ & $0(0.0 \%) / 45(100.0 \%)^{*}$ & $20(40.0 \%) / 30(60.0 \%)$ \\
\hline Total & $264(60.14 \%) / 124(28.25 \%)$ & $1(0.23 \%) / 432(98.41 \%)$ & $228(51.94 \%) / 160(36.45 \%)$ \\
\hline
\end{tabular}

Table 2. Estimated sensitivity and specificity for each pipeline for each antimicrobial. Sensitivity (A), specificity (B) values and their $95 \%$ confidence intervals (values between brackets) for each pipeline and each antibiotic. ${ }^{*}$ Average values do not include sensitivity for tigecycline. Cells with values greater or equal than 0.95 have a green background, 0.90-0.49 orange background and smaller than 0.9 red background. Between brackets, is the name of the institute that run the pipeline.

A: 


\begin{tabular}{|c|c|c|c|c|c|c|}
\hline Sensitivity & $\begin{array}{l}\text { Seqfinder/Abricate } \\
\text { (APHA) }\end{array}$ & $\begin{array}{l}\text { GeneFinder } \\
\text { (PHE) }\end{array}$ & $\begin{array}{l}\text { BLAST } \\
\text { (WBVR) }\end{array}$ & $\begin{array}{l}\text { ResFinder/PointFinder } \\
\text { (NVI) }\end{array}$ & $\begin{array}{l}\text { ARIBA } \\
\text { (UCM) }\end{array}$ & Average \\
\hline Ampicillin & $0.94(0.911,0.961)$ & $\begin{array}{l}0.971 \\
(0.949,0.986)\end{array}$ & $\begin{array}{l}0.934 \\
(0.905,0.957)\end{array}$ & $0.955(0.93,0.974)$ & $\begin{array}{l}0.974 \\
(0.952,0.987)\end{array}$ & 0.955 \\
\hline Azithromycin & $\begin{array}{l}0.945 \\
(0.866,0.985)\end{array}$ & $\begin{array}{l}0.932 \\
(0.847,0.977)\end{array}$ & $\begin{array}{l}0.877 \\
(0.779,0.942)\end{array}$ & $0.932(0.847,0.977)$ & $\begin{array}{l}0.932 \\
(0.847,0.977)\end{array}$ & 0.923 \\
\hline Cefotaxime & $\begin{array}{l}0.898 \\
(0.855,0.932)\end{array}$ & $\begin{array}{l}0.891 \\
(0.847,0.925)\end{array}$ & $\begin{array}{l}0.887 \\
(0.842,0.922)\end{array}$ & $0.906(0.864,0.938)$ & $\begin{array}{l}0.891 \\
(0.847,0.925)\end{array}$ & 0.894 \\
\hline Ceftazidime & $\begin{array}{l}0.946 \\
(0.909,0.971)\end{array}$ & $\begin{array}{l}0.938 \\
(0.899,0.965)\end{array}$ & $\begin{array}{l}0.933 \\
(0.894,0.961)\end{array}$ & $0.95(0.914,0.974)$ & $\begin{array}{l}0.933 \\
(0.894,0.961)\end{array}$ & 0.940 \\
\hline Chloramphenicol & $0.947(0.889,0.98)$ & $\begin{array}{l}0.947 \\
(0.889,0.98)\end{array}$ & $\begin{array}{l}0.947 \\
(0.889,0.98)\end{array}$ & $0.947(0.889,0.98)$ & $\begin{array}{l}0.947 \\
(0.889,0.98)\end{array}$ & 0.947 \\
\hline Ciprofloxacin & $0.908(0.867,0.94)$ & $\begin{array}{l}0.931 \\
(0.894,0.959)\end{array}$ & $\begin{array}{l}0.885 \\
(0.841,0.921)\end{array}$ & $0.935(0.898,0.962)$ & $\begin{array}{l}0.305 \\
(0.25,0.365)\end{array}$ & 0.793 \\
\hline Colistin & $\begin{array}{l}0.704 \\
(0.498,0.862)\end{array}$ & $\begin{array}{l}0.704 \\
(0.498,0.862)\end{array}$ & $\begin{array}{l}0.704 \\
(0.498,0.862)\end{array}$ & $0.704(0.498,0.862)$ & $\begin{array}{l}0.704 \\
(0.498,0.862)\end{array}$ & 0.704 \\
\hline Gentamicin & $\begin{array}{l}0.939 \\
(0.871,0.977)\end{array}$ & $\begin{array}{l}0.959 \\
(0.899,0.989)\end{array}$ & $\begin{array}{l}0.949 \\
(0.885,0.983)\end{array}$ & $0.959(0.899,0.989)$ & $\begin{array}{l}0.847 \\
(0.76,0.912)\end{array}$ & 0.931 \\
\hline Meropenem & $1.0(0.927,1.0)$ & $\begin{array}{l}1.0 \\
(0.927,1.0)\end{array}$ & $\begin{array}{l}1.0 \\
(0.927,1.0)\end{array}$ & $1.0(0.927,1.0)$ & $\begin{array}{l}1.0 \\
(0.927,1.0)\end{array}$ & 1.000 \\
\hline Nalidixic Acid & $\begin{array}{l}0.904 \\
(0.854,0.941)\end{array}$ & $\begin{array}{l}0.934 \\
(0.89,0.965)\end{array}$ & $\begin{array}{l}0.934 \\
(0.89,0.965)\end{array}$ & $0.929(0.884,0.961)$ & $\begin{array}{l}0.03 \\
(0.011,0.065)\end{array}$ & 0.746 \\
\hline Sulfamethoxazole & $\begin{array}{l}0.767 \\
(0.713,0.814)\end{array}$ & $\begin{array}{l}0.892 \\
(0.85,0.925)\end{array}$ & $\begin{array}{l}0.861 \\
(0.815,0.899)\end{array}$ & $0.885(0.842,0.92)$ & $\begin{array}{l}0.892 \\
(0.85,0.925)\end{array}$ & 0.859 \\
\hline Tetracycline & $\begin{array}{l}0.973 \\
(0.946,0.989)\end{array}$ & $\begin{array}{l}0.985 \\
(0.962,0.996)\end{array}$ & $\begin{array}{l}0.955 \\
(0.922,0.976)\end{array}$ & $0.973(0.946,0.989)$ & $\begin{array}{l}0.981 \\
(0.956,0.994)\end{array}$ & 0.973 \\
\hline Tigecyline & $1.0(0.025,1.0)$ & $\begin{array}{l}1.0 \\
(0.025,1.0)\end{array}$ & $\begin{array}{l}1.0 \\
(0.025,1.0)\end{array}$ & $1.0(0.025,1.0)$ & $\begin{array}{l}1.0 \\
(0.025,1.0)\end{array}$ & 1.000 \\
\hline Trimethoprim & $\begin{array}{l}0.868 \\
(0.818,0.909)\end{array}$ & $\begin{array}{l}0.961 \\
(0.926,0.982)\end{array}$ & $\begin{array}{l}0.947 \\
(0.91,0.973)\end{array}$ & $0.952(0.915,0.976)$ & $\begin{array}{l}0.961 \\
(0.926,0.982)\end{array}$ & 0.938 \\
\hline Average* & 0.903 & 0.926 & 0.909 & 0.925 & 0.800 & 0.893 \\
\hline
\end{tabular}

B:

\begin{tabular}{|c|c|c|c|c|c|c|}
\hline Specificity & $\begin{array}{l}\text { Seqfinder/Abricate } \\
\text { (APHA) }\end{array}$ & $\begin{array}{l}\text { GeneFinder } \\
\text { (PHE) }\end{array}$ & $\begin{array}{l}\text { BLAST } \\
\text { (WBVR) }\end{array}$ & $\begin{array}{l}\text { ResFinder/Pointfinder } \\
\text { (NVI) }\end{array}$ & $\begin{array}{l}\text { ARIBA } \\
\text { (UCM) }\end{array}$ & Average \\
\hline Ampicillin & $\begin{array}{l}0.965 \\
(0.879,0.996)\end{array}$ & $\begin{array}{l}0.947 \\
(0.854,0.989)\end{array}$ & $\begin{array}{l}1.0 \\
(0.937,1.0)\end{array}$ & $0.947(0.854,0.989)$ & $\begin{array}{l}0.965 \\
(0.879,0.996)\end{array}$ & 0.965 \\
\hline Azithromycin & $\begin{array}{l}0.894 \\
(0.851,0.929)\end{array}$ & $\begin{array}{l}0.758 \\
(0.702,0.809)\end{array}$ & $\begin{array}{l}0.823 \\
(0.771,0.867)\end{array}$ & $0.77(0.714,0.819)$ & $\begin{array}{l}0.725 \\
(0.667,0.777)\end{array}$ & 0.794 \\
\hline Cefotaxime & $\begin{array}{l}0.931 \\
(0.882,0.964)\end{array}$ & $\begin{array}{l}0.925 \\
(0.875,0.959)\end{array}$ & $\begin{array}{l}0.948 \\
(0.904,0.976)\end{array}$ & $0.936(0.889,0.968)$ & $\begin{array}{l}0.942 \\
(0.896,0.972)\end{array}$ & 0.936 \\
\hline Ceftazidime & $\begin{array}{l}0.884 \\
(0.831,0.925)\end{array}$ & $\begin{array}{l}0.884 \\
(0.831,0.925)\end{array}$ & $\begin{array}{l}0.909 \\
(0.86,0.945)\end{array}$ & $0.894(0.842,0.933)$ & $\begin{array}{l}0.904 \\
(0.854,0.941)\end{array}$ & 0.895 \\
\hline Chloramphenicol & $\begin{array}{l}0.891 \\
(0.847,0.925)\end{array}$ & $\begin{array}{l}0.839 \\
(0.79,0.881)\end{array}$ & $\begin{array}{l}0.869 \\
(0.823,0.906)\end{array}$ & $0.777(0.723,0.825)$ & $\begin{array}{l}0.763 \\
(0.708,0.812)\end{array}$ & 0.828 \\
\hline Ciprofloxacin & $\begin{array}{l}0.898 \\
(0.843,0.938)\end{array}$ & $\begin{array}{l}0.744 \\
(0.673,0.807)\end{array}$ & $\begin{array}{l}0.665 \\
(0.59,0.734)\end{array}$ & $0.739(0.667,0.802)$ & $\begin{array}{l}0.784 \\
(0.716,0.842)\end{array}$ & 0.766 \\
\hline Colistin & $\begin{array}{l}0.995 \\
(0.983,0.999)\end{array}$ & $\begin{array}{l}0.998 \\
(0.987,1.0)\end{array}$ & $\begin{array}{l}0.993 \\
(0.979,0.998)\end{array}$ & $0.99(0.975,0.997)$ & $\begin{array}{l}0.993 \\
(0.979,0.998)\end{array}$ & 0.994 \\
\hline
\end{tabular}




\begin{tabular}{|c|c|c|c|c|c|c|}
\hline Gentamicin & $\begin{array}{l}0.844 \\
(0.801,0.881)\end{array}$ & $\begin{array}{l}0.844 \\
(0.801,0.881)\end{array}$ & $\begin{array}{l}0.835 \\
(0.792,0.873)\end{array}$ & $0.835(0.792,0.873)$ & $\begin{array}{l}0.818 \\
(0.772,0.857)\end{array}$ & 0.835 \\
\hline Meropenem & $\begin{array}{l}0.927 \\
(0.896,0.951)\end{array}$ & $\begin{array}{l}0.927 \\
(0.896,0.951)\end{array}$ & $\begin{array}{l}0.93 \\
(0.899,0.953)\end{array}$ & $0.93(0.899,0.953)$ & $\begin{array}{l}0.927 \\
(0.896,0.951)\end{array}$ & 0.928 \\
\hline Nalidixic Acid & $\begin{array}{l}0.979 \\
(0.947,0.994)\end{array}$ & $\begin{array}{l}0.937 \\
(0.892,0.967)\end{array}$ & $\begin{array}{l}0.753 \\
(0.685,0.812)\end{array}$ & $0.926(0.879,0.959)$ & $\begin{array}{l}0.979 \\
(0.947,0.994)\end{array}$ & 0.915 \\
\hline Sulfamethoxazole & $\begin{array}{l}0.931 \\
(0.862,0.972)\end{array}$ & $\begin{array}{l}0.931 \\
(0.862,0.972)\end{array}$ & $\begin{array}{l}0.941 \\
(0.875,0.978)\end{array}$ & $0.931(0.862,0.972)$ & $\begin{array}{l}0.911 \\
(0.838,0.958)\end{array}$ & 0.929 \\
\hline Tetracycline & $0.96(0.908,0.987)$ & $\begin{array}{l}0.927 \\
(0.867,0.966)\end{array}$ & $\begin{array}{l}0.935 \\
(0.877,0.972)\end{array}$ & $0.919(0.857,0.961)$ & $\begin{array}{l}0.911 \\
(0.847,0.955)\end{array}$ & 0.931 \\
\hline Tigecyline & $\begin{array}{l}0.933 \\
(0.905,0.955)\end{array}$ & $\begin{array}{l}0.933 \\
(0.905,0.955)\end{array}$ & $\begin{array}{l}0.942 \\
(0.916,0.962)\end{array}$ & $0.933(0.905,0.955)$ & $\begin{array}{l}0.933 \\
(0.905,0.955)\end{array}$ & 0.935 \\
\hline Trimethoprim & $0.994(0.966,1.0)$ & $\begin{array}{l}0.975 \\
(0.937,0.993)\end{array}$ & $\begin{array}{l}0.975 \\
(0.937,0.993)\end{array}$ & $0.975(0.937,0.993)$ & $\begin{array}{l}0.975 \\
(0.937,0.993)\end{array}$ & 0.979 \\
\hline Average & 0.930 & 0.898 & 0.894 & 0.893 & 0.895 & 0.902 \\
\hline
\end{tabular}

Table 3. Questionnaire and average scores from the 9 collaborators for each of the pipelines evaluated. Interpretation questionnaire scores. Scores range from 1 being the lowest (worst) score to 5 being the highest (most positive) score. Between brackets, the name of the institute that run the pipeline.

(range 1 to 5.1 being the lowest score and 5 the highest score)

$\begin{array}{lllll}\text { Seqfinder/Abricate } & \text { Genefinder. } & \text { BLAST } & \text { ResFinder/ } & \text { ARIBA } \\ \text { (APHA) } & \text { (PHE) } & \text { (WBVR) } & \text { PointFinder } & \text { (UCM) } \\ & & & (\mathrm{NVI}) & \end{array}$

\begin{tabular}{|c|c|c|c|c|c|}
\hline 1. First impressions when open the results table. & 4.22 & 4 & 4.22 & 3.33 & 2.11 \\
\hline 2. How easy was it to find your results in the output file? & 4.44 & 4.44 & 4.33 & 4 & 2.56 \\
\hline $\begin{array}{l}\text { 3. How easy did you find it to link the gene/mutation to } \\
\text { your phenotype? }\end{array}$ & 4.06 & 3.89 & 4 & 3.78 & 2.78 \\
\hline $\begin{array}{l}\text { 4. How a non-related to the subject individual will find } \\
\text { understanding the outputs? }\end{array}$ & 3.44 & 3 & 3.67 & 3.33 & 1.78 \\
\hline 5. Availability of QC metrics such as mean coverage, etc & 4.78 & 4.22 & 1.22 & 3.11 & 4 \\
\hline 6. Time used to extract the information & 4.11 & 4.22 & 3.78 & 3.44 & 2.44 \\
\hline 7. What is your preferred pipeline? & 3.78 & 4.11 & 3.44 & 3.67 & 2.33 \\
\hline Average & 4.12 & 3.98 & 3.52 & 3.52 & 2.57 \\
\hline
\end{tabular}

\section{Figures}




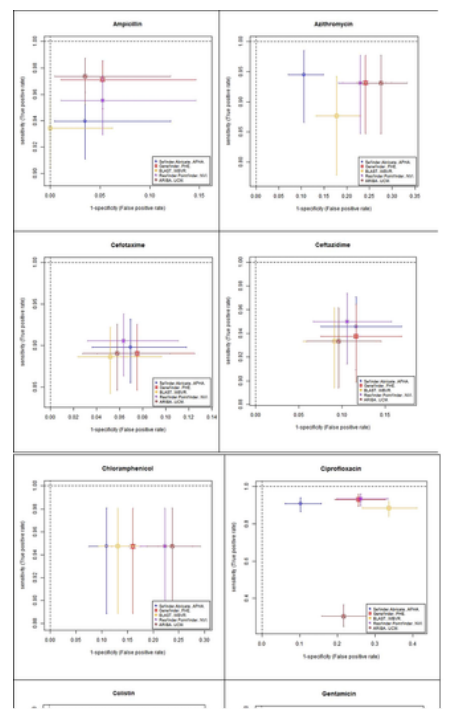

\section{Figure 1}

Each graph represents the sensitivity and specificity values (and $95 \%$ confidence intervals) for each antimicrobial detected by the 5 pipelines on the receiver operating characteristic (ROC) coordinate system. Since there are no isolates resistance to tigecycline, its sensitive value is equal to 1 for all the pipelines. 